\title{
分析 GPS 在工程测量中的主要应用
}

\section{Analysis of the Application of GPS in Engineering Survey}

\section{陈育}

Yu Chen

贵州宇宏达测绘有限公司

中国・贵州 遵义 563000

Guizhou Yu Hongda Surveying and Mapping Co.,

Ltd.,

Zunyi, Guizhou, 563000, China
【摘 要】论文就 GPS 测量技术在工程测量工作中的优势特点、GPS 测量技术流程以及 GPS 在工程测量中的主要应用方面进行了相关讨论, 希望对提高中国工程测量工作的有 效性,促进中国工程行业的发展等方面起到一定的借鉴。

【Abstract】This paper discusses the advantages and characteristics of GPS survey technology in engineering survey, the process of GPS survey technology and the main application of GPS in engineering survey, hoping to improve the effectiveness of China's engineering survey and promote the development of China's engineering industry.

【关键词】GPS 测量技术; 工程测绘; 优势特点

【Keywords】GPS surveying technology; engineering surveying and mapping; advantage characteristic

【DOI】10.36012/se.v2i1.1174

\section{1 引言}

将 GPS 测量技术应用到中国的工程测量工作中, 可以有 效提高工程测量的水平，尤其是体现在地形以及土地的测量 工作中,其应用效果更佳。因此,中国相关的工程测绘单位需 要加强对 GPS 测量技术的分析, 明确其在工程测量工作中的 应用优势,进而实现 GPS 测量技术的更好应用。

\section{GPS 测量技术在工程测量中的优势}

\section{1 功能性较强, 测绘范围较广}

GPS 测绘技术在工程测量工作中的首要优势就是功能性 较强, 且测绘范围相对较广。中国传统的工程测绘技术, 在实 际工程测量工作中, 尤其是在较长距离的测量工作中, 不仅需 要消耗较多的人力, 而且在测量效率和测量精度上也相对较 低, 而应用 GPS 测绘技术则可以很好地弥补这个缺陷。通过 应用 GPS 测绘技术进行工程测量工作时，当基线在 $20 \mathrm{~km}$ 以 内时, GPS 测绘技术的精度也可以保持在 $1 \times 10^{-6} \sim 2 \times 10^{-6}$; 当基 线距离在 100 500km 时,GPS 测绘技术则可以将测量精度保 持在 $10^{-6} \sim 10^{-7}$ 。基于 GPS 测绘技术的这个优势特点, 其在中 国的航空、大地、工程等各个领域中的应用也越来越广泛。

\section{2 定位的精准度较高}

GPS 测绘技术可以分为动态测绘和静态测绘, 这两种测 绘方式的测量精度都较高。GPS 测绘技术从测量原理上来看, 主要是利用太空中的三个卫星来进行定位的, 然后在定位的
基础上再对地球上的某一个位置进行可靠的测量, 最终形成 空间星座、地面控制以及用户设备等三个部分, 共同完成工程 测量工作。因此, GPS 测绘技术在实际的工程测量上更加便捷 和高效,可以为工作人员提供完善的测量信息,在一定意义上 也可以实现全天性的自动化测量。

\section{GPS 测量技术流程}

GPS 测量技术流程分析见表 1 。

表 1 GPS 测量技术流程

\begin{tabular}{c|c}
\hline 主要流程 & \multicolumn{1}{|c}{ 具体分析 } \\
\hline 定位测量点 & $\begin{array}{c}\text { 定位原则是以安全便捷为前提, 工作人员需要选择比 } \\
\text { 响 GP阔的环境中进行作业。另外, 还需要避免一切会影 } \\
\text { GPS 的测量点, 作为以后工程测绘的依据 }\end{array}$ \\
\hline 构建测量标点 & $\begin{array}{l}\text { 在定位好测量点后, 工作人员要在其位置上安装测量 } \\
\text { 一测量标志会随着工程测绘环境的不同而不同, }\end{array}$ \\
\hline 测量观测 & $\begin{array}{c}\text { 工作人员需要利用 GPS 测量技术进行室外的观测, 从 } \\
\text { 而接收卫星定位系统传达的数据, 并对其进行分析 }\end{array}$ \\
\hline
\end{tabular}

\section{GPS 在工程测量中的主要应用}

\subsection{GPS 测量技术在城市建设中的应用}

针对中国城市的不同导线级别划分, 工作人员需要开展 不同的控制点的设置, 而通过应用 GPS 测量技术, 可以有效 提高测量工作的准确度和测量效率。其实际应用过程如下: 首 
先, 工作人员需要在城市配套设施建设中增加和 GPS 测量技 术相关的城市控制测量设备，为后期的工程测量工作奠定坚 实的基础; 其次,工作人员在正式开始应用 GPS 测量技术进 行工程测绘之前, 需要先对地形图中的一些细微部分进行详 细的测绘, 从而为后期的地形勘探工作提供科学的参考数据; 最后, 对于环境较为恶劣的工程测量, 工作人员还需要增加优 化设备, 以此来保证测绘城市的顺利进行 ${ }^{[2]}$ 。

\subsection{GPS 测量技术在监测工程中的应用}

工程在实际建设过程中, 经常会因为各种人为或者是环 境方面的因素出现地形或者是建筑物的变形,在这种情况下, 应用中国传统的工程测绘方法很难对其进行测量, 而通过应 用 GPS 测量技术, 工作人员则可以进行精准的三维定位, 因 此, 在很大程度上也可以强化所监测工程的细节。

另外, 随着中国 GPS 测量技术的不断发展完善, 其在静 态系统的定位精度上也有所提高, 其在实际的工程测量工作 中, 基线精度也从 $10^{-7}$ 提升到了 $10^{-6}$ 。在动态测量方面, 精度 也已经突破了厘米级别, 因此可以满足工程监测的需要。

\subsection{GPS 测量技术在水下地形测绘中的应用}

中国传统的测绘技术在进行水下地形测绘工作时, 主要
是通过探测仪所发射出的超声波来实现对水深的测量，或者 是应用潮位仪进行测量, 然后再对所测量的数据进行运算以 后, 最终得出水下地形的高度。而通过应用 GPS 测量技术, 可 以实现和潮位仪、终端设备联合实验, 从而完善水下测绘工作 的系统, 提高水下地形测绘工作的精确度 ${ }^{[3]}$ 。

\section{5 结语}

总而言之, GPS 测量技术和中国传统的工程测绘技术相 比较, 具有很多的测量优势, 具体表现为功能性较强, 且测绘 范围较广以及定位的精准度较高。因此, 在中国城市建设、监 测工程以及水下地形测绘工作中的应用也较为广泛，在很大 程度上也提升了测量精确度, 故而, 其应用前景也较为广泛。

\section{参考文献}

[1]赵爽.GPS 测量技术在工程测量中的应用 [J].工程技术研究, 2017(12):74-75.

[2]胡永兴.GPS 在工程测量中的应用研究 [J].工程建设与设计, 2017(12):5-6.

[3]张卫.GPS 测量技术在工程测量中的应用研究[J].建设科技, 2017(21):120-121.

\section{(上接第 25 页)}

与此同时也是环环相扣、缺一不可的。那么煤田三维地震勘探 的工作就需要将这三部分工作一体化, 在采集高质量数据的 基础上, 高效、准确、及时地完成数据处理及资料解释的工作, 只有很好地完成这一系列的工作。

\section{1 矿井周围的地震数据资料采集工作}

煤田三维地震勘探测量是为了确定埋设位置做准备, 开 孔就是为了埋藏炸药。基本的工作流程是地震波在遇到岩层 界面之后会反射回来, 之前埋藏好的检波器会接收到这些地 震波, 并通过之前布的线传回到仪器车上, 仪器车上的工具会 将传回来的信号保存下来, 这就得到了矿井周围的地震数据 资料。

\section{2 所得到的地震数据的处理工作}

所得到的地震数据的处理工作所说的是把矿井周围的地 震数据资料采集并输入到数据处理的电子计算机。然后根据 各不相同的要求, 使用各不相同的工具将这些数据进行分析、 处理。再然后将这些数据进行归类编排, 重点展示出有效波信 号,删除或者隐藏那些干扰的没有用的信号。最后这些经过处 理的不同数据经过叠加形成三维的数据体, 从而方便相关人 员得到不同的图。

\section{3 处理后的地震资料解释工作}

经过处理的地震数据再经过加工, 最终变成地质成果, 这 个过程就是我们所说的处理后的地震资料解释工作过程。在 这项工作中相关人员需要熟知波动理论以及地质、地表以及 钻矿井和其他各种知识, 以这些知识为基础, 与之前所得到地 震书记进行分析研究, 对所开矿区域地质、岩层以及岩性做出 分析并解释给相关的人员解释, 最终将这些制成文件, 从而为 煤田的开采工作打好基础。

\section{5 结语}

煤田矿井开拓及开采是煤炭行业很重要的一部分工作内 容,随着科技的不断发展, 三维地震勘探技术也广泛地应用在 煤田矿井开拓及开采中, 这些新技术的应用可以促进中国煤 炭行业的进步，也可以大大减少煤田矿井开拓及开采中的不 安全因素, 为促进中国煤炭行业的发展做出贡献, 希望本文对 相关领域的研究有借鉴意义。

\section{参考文献}

[1]杨双安,张胤涁, 许鸿雁.煤田三维地震勘探技术的应用及发展 前景[J].物探与化探,2004(6):500-503.

[2]刘芳晓.三维地震勘探技术在山东巨野煤田某井田的应用[J]. 西部探矿工程,2017(11):130-132. 